\title{
A missed opportunity: underutilization of inpatient behavioral health services to reduce injection drug use sequelae in Florida
}

\author{
Austin E. Coye ${ }^{1 *}$ (D, Mackenzie T. Jones ${ }^{1}$, Kasha J. Bornstein ${ }^{1}$, Hansel E. Tookes ${ }^{2}$ and Joan E. St. Onge ${ }^{3}$
}

\begin{abstract}
Background: People who inject drugs (PWID) suffer high morbidity and mortality from injection related infections (IRI). The inpatient setting is an ideal opportunity to treat underlying substance use disorder (SUD), but it is unclear how often this occurs.

Objectives: To quantify the utilization of behavioral health senvices for PWID during inpatient admissions for IRI.

Methods: Data for all hospital admissions in Florida in FY2017 were obtained from the Agency for Healthcare Administration. Hospitalization for IRI were obtained using a validated ICD-10 algorithm and treatment for substance use disorder was quantified using ICD-10-Procedure Coding System (ICD-10-PCS) codes.

Result: Among the 20,001 IRI admissions, there were 230 patients who received behavioral health services as defined by ICD-10PCS SAT codes for treatment for SUD.

Conclusions: In a state with a large number of IRI, only a very small portion of admissions received behavioral health services. Increased efforts should be directed to studying referral patterns among physicians and other providers caring for this population and increasing utilization of behavioral health services.
\end{abstract}

Keywords: People who inject drugs, Opioids, MOUD, Inpatient addiction services, Addiction

\section{Background}

People who inject drugs (PWID) are a vulnerable patient population who often require recurrent hospitalization for sequelae of drug use [1-4]. This vulnerable population experiences increased mortality risk after hospitalization as well as high risk for readmission [5]. A national 2016 analysis found approximately $24 \%$ of patients hospitalized for opioid overdose had at least one readmission within 90 days of discharge and 3\% were readmitted for overdose [6]. The high rehospitalization rates in this population signify a need for more effective medical care during hospital

\footnotetext{
* Correspondence: austincoye@gmail.com

'University of Miami Miller School of Medicine, 1600 NW 10th Ave \#1140, Miami, FL 33136, USA

Full list of author information is available at the end of the article
}

stays and provide critical windows of opportunity for hospital physicians to engage these patients in treatment for underlying substance use disorder(s) (SUD).

Significant recent evidence demonstrates myriad benefits of initiating treatment for underlying SUD in inpatient settings [7-12]. Peterson et al. found that identifying patients with SUD while in-hospital is an effective method to target prevention services that reduce opioid-related morbidity and mortality [12]. Wakeman et al. demonstrated that implementation of an inpatient addiction consult team resulted in decreased admissions for patients with SUD [8]. Kimmel et al. determined treatment with medication for opioid use disorder (MOUD) following hospitalization for injection drug use-associated infective endocarditis reduced mortality during the month that MOUD was received [7]. Despite the evidence that initiation 
of treatment for SUD during hospital admissions reduces morbidity and mortality in this population, previous literature indicates that these services are likely underutilized [13, 14]. However, the extent to which addiction services are utilized in inpatient settings is not well known.

The aim of this large-scale study was to assess utilization of addiction services during the hospitalization of 20,001 PWID admitted for injection related infection (IRI) across every Florida hospital during Fiscal Year 2017 (FY2017).

\section{Methods}

We conducted a retrospective review of patients hospitalized for IRI in Florida during FY2017, using the Agency for Health Care Administration (AHCA) Hospital Inpatient Limited Data Set. AHCA disclaims responsibility for analysis, interpretations, and conclusions. PWID admissions for IRI were identified using an algorithm combining International Classification of Diseases, 10th Revision (ICD-10) codes indicating drug use (opioids, cocaine, amphetamine, overdose, other psychoactive); and common infectious sequelae (endocarditis, osteomyelitis, bacteremia-and/or-sepsis, and skin-and-soft-tissue infections (SSTI)). The algorithm was validated using British Columbia Hepatitis Testers Cohort data and noted to have a sensitivity of $63 \%$ and a specificity of $100 \%$ [15].

PWID admissions between 18 and 75 years of age and with length of stay (LOS) $<60$ days were included. Patients who expired during hospitalization were excluded. Age and LOS restrictions were included to increase specificity of the PWID identification algorithm and patients that expired during the stay were excluded because they may have been considered too sick to receive inpatient treatment of SUD [16].

ICD-10-Procedure Coding System codes were used to assess treatment for SUD. These are labelled in ICD-10 as codes for substance abuse treatment, however we will refer to them as Substance Addiction Treatment (SAT) to avoid the use of stigmatizing language as outlined by the National Institute on Drug Abuse [17]. Codes for SAT (PCS SAT) included detoxification services, individual counseling, group counseling, medication management, and pharmacotherapy. Individual and group counseling modalities included cognitive, behavioral, cognitive-behavioral, 12-step, interpersonal, vocational, psychoeducational, motivational enhancement, confrontational, continuing care, spiritual and pre/post-test infectious disease. Individual psychotherapy included cognitive, behavioral, cognitive-behavioral, 12-step, interpersonal, interactive, psychoeducation, motivational enhancement, confrontational, supportive, psychoanalysis, psychodynamic, and psychophysiological. Medication management and pharmacotherapy for SAT comprised nicotine replacement, methadone, levo-alpha-acetylmethadol, disulfiram, naltrexone, naloxone, clonidine, bupropion, psychiatric medication, and nicotine replacement medications.

\section{Results}

This analysis includes 20,001 PWID admitted to Florida hospitals during FY2017 for IRI. Admissions most often included bacteremia-and/or-sepsis (53\%) followed by SSTI (48\%). Opioids were the most common drug recorded (52\%). The majority (55\%) of patients had publicly subsidized insurance, while $33 \%$ were uninsured. Median LOS was nine days (IQR: 3-12). (Appendix).

Among the 20,001 PWID admissions included, there were 230 patients who received behavioral health services as defined by PCS SAT, representing fewer than $2 \%$ of all PWID admissions. Among these admissions, female patients comprised $44 \%$. Most $(n=200)$ were identified as non-Hispanic. The majority $(n=196)$ were identified as white. The most common service rendered was detoxification $(n=220)$, followed by Individual Psychotherapy for SAT (31 admissions). Only 29 of 20, 001 admissions recorded medication management or pharmacotherapy for SAT (including methadone, naltrexone, and/or clonidine) during the study period (Table 1).

\section{Discussion}

The data presented reveal that referral to addiction services and prescription of SAT medications for hospitalized PWID are underutilized in Florida. The median LOS was nine days among all PWID admissions, indicating ample time for SAT initiation. Data from this statewide sample demonstrates significant opportunities to increase utilization of these services, potentially reducing readmission rates, mitigating preventable toxicological and infectious morbidity, and reducing mortality. Previous studies demonstrate that PWID admissions come at great social and economic cost to Florida and nationwide $[15,18]$. Limited billing for PCS-SAT modalities through analysis of billing and coding records in this statewide sample suggests major gaps exist during inpatient treatment of underlying SUD $[15,19]$.

Other studies suggest similar deficiencies in implementation of best practices for hospitalized PWID. A national survey of hospitalists indicated that while $84.5 \%$ reported "often or always" caring for patients with opioid use disorder (OUD), $88.9 \%$ "rarely or never" initiated buprenorphine in the inpatient setting [20]. A 2019 analysis of Veterans Health Administration data found only $16 \%$ of veterans began any SUD treatment following initial diagnosis [13]. Only $2.7 \%$ began MOUD within 14 days of their first encounter [13]. In a retrospective review of patients hospitalized with injection drug use-associated infective endocarditis between 2004 and 2014, Rosenthal et al. found fewer than $25 \%$ of PWID received psychiatry or addiction medicine consultation [14]. Addiction was mentioned in only $56 \%$ of discharge summary plans and only $7.8 \%$ of patients had planning for MOUD [14]. Retrospective analysis also found that over $25 \%$ of those patients were deceased by 2016 and the median age at death was 40.9 years [14]. While our findings are similar to others, this study uses a unique algorithm to quantify SAT in a large inpatient 
Table 1 Demographics of PWID Admissions who received ICD-10-PCS Substance Addiction Treatment

\begin{tabular}{|c|c|}
\hline & Inpatient Admissions (\%) \\
\hline \multicolumn{2}{|l|}{ Biological Sex } \\
\hline Male & $128(56)$ \\
\hline Female & $102(44)$ \\
\hline \multicolumn{2}{|l|}{ Ethnicity } \\
\hline Hispanic or Latino & $25(11)$ \\
\hline Non-Hispanic or Latino & $200(87)$ \\
\hline Other & $5(2.0)$ \\
\hline \multicolumn{2}{|l|}{ Race } \\
\hline Black or African American & $20(8.7)$ \\
\hline White & $196(85)$ \\
\hline Other & $14(6.1)$ \\
\hline \multicolumn{2}{|l|}{ Age (years) } \\
\hline $18-29$ & $40(17)$ \\
\hline $30-39$ & $68(30)$ \\
\hline $40-49$ & $62(27)$ \\
\hline $50-59$ & $37(16)$ \\
\hline $60-75$ & $23(10)$ \\
\hline Mean Age (years) & 40.7 \\
\hline \multicolumn{2}{|l|}{ Insurance Status } \\
\hline Federal & $47(20)$ \\
\hline State, County, Local & $57(25)$ \\
\hline Uninsured & $41(18)$ \\
\hline Private Insurance & $83(36)$ \\
\hline Other & $2(0.9)$ \\
\hline \multicolumn{2}{|l|}{ Discharge Status } \\
\hline Discharge or transferred & $193(84)$ \\
\hline Discharged to Hospice & $2(0.9)$ \\
\hline Left AMA & $35(15)$ \\
\hline \multicolumn{2}{|l|}{ Hospital Service Utilization } \\
\hline Median Length of Stay & 6 days \\
\hline Substance Addiction Treatment ${ }^{a}$ & Admissions (\%) \\
\hline Connection to any Addiction Services & $230(100)$ \\
\hline Detoxification Services & $220(96)$ \\
\hline Individual or Group Counseling & 31 (13) \\
\hline Medication Management or Pharmacotherapy & 29 (13) \\
\hline
\end{tabular}

${ }^{\mathrm{a} A d m i s s i o n s ~ c o u l d ~ i n c l u d e ~ m o r e ~ t h a n ~ o n e ~ I C D-P C S ~ C o d e ~}$

sample, one that can replicated easily in other states to monitor treatment and outcomes of initiatives to increase SAT.

A major limitation of this study is the structure of ICD-10 coding for drug use disorders and SAT. To date, the ICD-10 lacks specific code(s) for PWID, complicating efforts to study the care of this patient population and necessitating algorithmic approaches to identification of hospitalized PWID [15, $21,22]$.While highly specific, the statistical algorithm used in this study underestimates the real number of hospitalized PWID in Florida during the study period. In addition to built-in stigmatizing language such as "abuse", there is no specific category for major medications such as buprenorphine or acamprosate and it includes vague categories such as "psychoactive medication." ICD-10-PCS codes for SAT pharmacotherapy include drugs such as nicotine replacement therapy and bupropion, which have no known utility in the medication-assisted treatment of opioid use. Additionally, levo-alpha-acetylmethadol is not used in the United States. Utilization of ICD-10-PCS codes risks possible lack of coding, miscoding, or underreporting of services rendered. Although this methodology does not capture all psychiatric services rendered to hospitalized PWID, it is consistent with other reports and suggests general underutilization deserving of further exploration. 
While we did not capture brief counseling about SUD or other informal counseling during inpatient stay, brief counseling is still likely underutilized. One study observed that $22 \%$ of hospitalized patients seen by an addiction consultation-liaison service received only brief counseling without formal referral and without essential risk mitigation modalities [23]. Brief counseling is not as effective as other interventions. In a randomized trial comparing in-hospital MOUD initiation versus brief counseling, MOUD was associated with increased engagement in treatment and reduced illicit opioid use [24]. Patients may also have been referred to outpatient behavioral health treatment or to harm reduction organizations, neither of which was captured in this study.

One proposed explanation for the paucity of interventions offered to PWID may be presumed lack of social stability or resources for treatment adherence. However, a Swedish study found parity in 12-month retention rates among MOUD program participants with and without strict social stability requirements [25]. Another study found homelessness positively predicted MOUD initiation among hospitalized adults seen by an addiction consult service, indicating that hospitalization may be an important opportunity to engage typically harder to reach populations [26]. Insurance status was also likely not the primary barrier in receiving behavioral health services as only $36 \%$ of the admitted patients receiving services in that study had private insurance.

The latter years of the 2010s saw the first prolonged, multiyear decline in average life expectancy in the United States in over a century, secondary to complications of hazardous opioid use [27]. This mortality rate has amplified considerably in the setting of the COVID19 pandemic: early results indicate national Emergency Medical Services activations for opioid-related cardiac arrests in 2020 occurred 48.5\% above baseline [28]. This dramatic worsening of preventable opioid morbidity and mortality emphasizes the critical imperative of adapting evidencebased best practices for addiction during hospitalization.

The American College of Physicians, the National Institutes of Health, and the Infectious Diseases Society of America have issued calls for action to implement and scale up effective SUD treatment in healthcare settings [29-31]. The attendees of a National Academies of Sciences, Engineering, and Medicine workshop on "Integrating Infectious Disease Considerations with Response to the Opioid Epidemic" proposed an action plan that includes screening for OUD in all relevant settings and immediate prescription of effective medication for OUD and/or opioid withdrawal symptoms [32]. In an article on the diagnosis and management of OUD in hospitalized patients, Herscher et al. emphasize the roles that hospitalists play in engaging patients with OUD in treatment [33]. Writing on the management of co-occurring OUD and infectious disease in inpatient settings, Eaton and Vettese echo the importance of initiating MOUD for patients experiencing infectious sequelae, noting that management of injection-related infections "is incomplete without addressing the underlying [SUD]." The authors recommend a number of initiatives focused on reducing stigma, addiction medicine consultation, improved pain management, and implementing harm reduction resources [34].

While the findings of the present study highlight the underutilization of services to address SUD in hospitalized patients, these guidelines and calls for action have the potential to enhance the role hospitalists play in the treatment of PWID. However, hospitalists cannot achieve these goals alone; other systemic and structural changes are necessary to improve high-quality SUD treatment access. The undersupply of addiction services likely contributes to low SUD service utilization. Involvement of inpatient psychiatry and addiction teams are effective means to reduce addiction severity and substance use, initiate inpatient care, and transition to outpatient care for SUD [10,35]. A study of patients with SUD admitted for serious infection demonstrated addiction medicine consultation improved rates of SUD treatment, increased likelihood of completion of antimicrobial therapy, and reduced readmission rates [9]. However, a national survey of hospitalists found that only $67.9 \%$ of respondents reported access to addiction specialists. Those with access to addiction specialists were 4.4 times more likely to screen and three times more likely to refer patients for treatment than those without [20]. Unfortunately, the pipeline for physicians training in addiction medicine or addiction psychiatry is low-flow; during 2020-21 in Florida, only 13 physicians trained in accredited programs in these specialties [36]. Prioritizing expansion of these oneyear training programs by including them in state and federal graduate medical education funding expansion could rapidly increase availability of addiction specialists. This lack of specialists also likely drives a paucity of outpatient services, presenting additional barriers for transitions of care of SUD is started inpatient [37, 38]. Education on SUD and MOUD has been a focus in Florida since 2019, with the call for and support of education programs at medical schools focusing on SUD, MOUD and opportunities for intervention [39]. Institutional establishment and/or partnerships with syringe access programs, expansion of provider education for OUD and elimination of procedural barriers including the "X-Waiver" and prior authorizations are other ways to reduce major treatment gaps [40].

\section{Conclusion}

In a state with a large number of IRI, few PWID received behavioral health services, highlighting opportunities to address risk factors for increased morbidity and mortality. Enhanced funding for addiction specialists and harm reduction organizations, increased SUD education, and reduction of barriers to prescribing MOUD will support our hospital-based colleagues in their efforts to expand access to treatment in this population. 


\section{Appendix}

Table 2 PWID Hospitalizations for Infections in Florida, FY 2017

\begin{tabular}{ll}
\hline Characteristic & PWID Admissions (\%) \\
\hline Biological Sex & \\
Male & $10,779(54)$ \\
Female & $9222(46)$ \\
Ethnicity & \\
Hispanic or Latinx & $1608(8)$ \\
Non-Hispanic or Latinx & $17,930(90)$ \\
Unknown & $299(2)$
\end{tabular}

\section{Race}

Black or African American

White

$16,694(84)$

Other

$862(4.4)$

\section{Age (years)}

$<29$

30-39

40-49

50-59

60-75

Mean Age (years)

44.5 (IQR 33-56)

\section{Insurance Status}

Federal

Admissions (\%)

5625 (28)

State, County, Local

$5045(25)$

Uninsured

Private Insurance

Other

\section{Length of Stay (Days)}

9.39 (IQR 3-12)

\section{Discharge Status}

Discharge or transferred

Expired

Left AMA

\section{Infection Type ${ }^{a}$}

Skin and Soft Tissues

Osteomyelitis

Bacteremia/Sepsis

Endocarditis

$1908(10)$

aMany admissions had more than one infection diagnosis coded per admission

\section{Abbreviations}

PWID: People Who Inject Drugs; IRI: Injection Related Infections; SUD: Substance Use Disorder; ICD-10 PCS: International Classification of Diseases, 10th Revision, Procedure Coding System; SAT: Substance Addiction Treatment; PCS SAT: Procedure Coding System Codes for Substance Addiction Treatment; MOUD: Medication for Opioid Use Disorder; FY2017: Fiscal Year 2017; AHCA: Agency for Health Care Administration; SST: Skin and Soft Tissue Infection; LOS: Length of Stay; OUD: Opioid Use Disorder

\section{Acknowledgements}

The authors would like to thank the Infectious Diseases Society of America Foundation for support in this investigation via the Medical Scholars Program and the Miami Center for AIDS Research for statistical assistance.

\section{Conflict of interest}

The authors have no COI to report.

\section{Sources of support}

Infectious Diseases Society of America Foundation.

The Miami Center for AIDS Research.

\section{Authors' contributions}

All authors contributed to the design and interpretation of the study. AC and MJ analyzed the data. AC, MJ, and KB substantially contributed to the manuscript and all authors read and approved the final version to be published.

\section{Funding}

Research was partially financially supported by the Infectious Disease Society of America.

\section{Availability of data and materials}

The data that support the findings of this study are available from Agency for Health Care Administration but restrictions apply to the availability of these data, which were used under license for the current study, and so are not publicly available. Data are however available from the authors upon reasonable request and with permission of Agency for Health Care Administration.

\section{Declarations}

Ethics approval and consent to participate

The study was approved by the University of Miami Institutional Review Board (IRB \#20180242).

\section{Consent for publication}

Not applicable.

\section{Competing interests}

The authors declare that they have no competing interests.

\section{Author details}

${ }^{1}$ University of Miami Miller School of Medicine, 1600 NW 10th Ave \#1140, Miami, FL 33136, USA. ²Department Medicine, Division of Infectious Diseases, University of Miami Miller School of Medicine, Miami, FL 33136, USA.

${ }^{3}$ Department of Medicine, Division of General Internal Medicine, University of Miami Miller School of Medicine, Miami, FL 33136, USA.

Accepted: 11 May 2021

Published online: 31 May 2021

\section{References}

1. Ronan MV, Herzig SJ. Hospitalizations related to opioid abuse/dependence and associated serious infections increased sharply, 2002-12. Health Aff (Millwood). 2016;35(5):832-7. https://doi.org/10.1377/hlthaff.2015.1424.

2. Zibbell JE, Asher AK, Patel RC, Kupronis B, labal K, Ward JW, et al. Increases in acute hepatitis $C$ virus infection related to a growing opioid epidemic and associated injection drug use, United States, 2004 to 2014. Am J Public Health. 2018;108(2):175-81. https://doi.org/10.2105/AJPH.2017.304132.

3. Wurcel AG, Anderson JE, Chui KK, et al. Increasing Infectious Endocarditis Admissions Among Young People Who Inject Drugs. Open Forum Infect Dis. 2016;3(3):ofw157.

4. McCarthy NL, Baggs J, See I, et al. Bacterial infections associated with substance use disorders, large cohort of United States hospitals, 2012-2017. Clin Infect Dis. 2020;71(7):e37-44. https://doi.org/10.1093/cid/ciaa008.

5. Walley AY, Paasche-Orlow M, Lee EC, Forsythe S, Chetty VK, Mitchell S, et al. Acute care hospital utilization among medical inpatients discharged with a substance use disorder diagnosis. J Addict Med. 2012;6(1):50-6. https://doi. org/10.1097/ADM.0b013e318231de51. 
6. Peterson C, Liu Y, Xu L, Nataraj N, Zhang K, Mikosz CA. U.S. national 90-day readmissions after opioid overdose discharge. Am J Prev Med. 2019;56(6): 875-81. https://doi.org/10.1016/j.amepre.2018.12.003.

7. Kimmel SD, Walley AY, Li Y, Linas BP, Lodi S, Bernson D, et al. Association of Treatment with Medications for opioid use disorder with mortality after hospitalization for injection drug use-associated infective endocarditis. JAMA Netw Open. 2020;3(10):e2016228. https://doi.org/10.1001/jama networkopen.2020.16228.

8. Wakeman SE, Kane M, Powell E, Howard S, Shaw C, Regan S. Impact of Inpatient Addiction Consultation on Hospital Readmission. J General Internal Med. 2020.

9. Marks LR, Munigala S, Warren DK, Liang SY, Schwarz ES, Durkin MJ. Addiction medicine consultations reduce readmission rates for patients with serious infections from opioid use disorder. Clin Infect Dis. 2019;68(11): 1935-7. https://doi.org/10.1093/cid/ciy924.

10. Trowbridge $P$, Weinstein $Z M$, Kerensky $T$, Roy $P$, Regan $D$, Samet $J H$, et al. Addiction consultation services - linking hospitalized patients to outpatient addiction treatment. J Subst Abus Treat. 2017;79:1-5. https://doi.org/10.101 6/j.jsat.2017.05.007

11. Wakeman SE, Metlay JP, Chang Y, Herman GE, Rigotti NA. Inpatient addiction consultation for hospitalized patients increases post-discharge abstinence and reduces addiction severity. J Gen Intern Med. 2017;32(8): 909-16. https://doi.org/10.1007/s11606-017-4077-z.

12. Peterson C, Xu L, Mikosz CA, Florence C, Mack KA. US hospital discharges documenting patient opioid use disorder without opioid overdose or treatment services, 2011-2015. J Subst Abus Treat. 2018;92:35-9. https://doi. org/10.1016/j.jsat.2018.06.008

13. Kraemer KL, McGinnis KA, Fiellin DA, et al. Low levels of initiation, engagement, and retention in substance use disorder treatment including pharmacotherapy among HIV-infected and uninfected veterans. J Subst Abus Treat. 2019;103:23-32. https://doi.org/10.1016/j.jsat.2019.05.007.

14. Rosenthal ES, Karchmer AW, Theisen-Toupal J, Castillo RA, Rowley CF. Suboptimal addiction interventions for patients hospitalized with injection drug use-associated infective endocarditis. Am J Med. 2016;129(5):481-5. https://doi.org/10.1016/j.amjmed.2015.09.024.

15. Coye AE, Bornstein KJ, Bartholomew TS, et al. Hospital Costs of Injection Drug Use in Florida. Clin Infect Dis. 2020.

16. Tookes H, Diaz C, Li H, Khalid R, Doblecki-Lewis S. A cost analysis of hospitalizations for infections related to injection drug use at a county safety-net hospital in Miami, Florida. PLoS One. 2015;10(6):e0129360. https:// doi.org/10.1371/journal.pone.0129360.

17. National Institute on Drug Abuse. Words Matter - Terms to Use and Avoid When Talking About Addiction. https://www.drugabuse.gov/nidamedmedical-health-professionals/health-professions-education/words-matterterms-to-use-avoid-when-talking-about-addiction. Updated October 28, 2020. Accessed 29 Dec 2020.

18. Hsu DJ, McCarthy EP, Stevens JP, Mukamal KJ. Hospitalizations, costs and outcomes associated with heroin and prescription opioid overdoses in the United States 2001-12. Addiction. 2017;112(9):1558-64. https://doi.org/1 $0.1111 /$ add.13795.

19. Blanchard J, Weiss AJ, Barrett ML, Stocks C, Owens PL, Coffey R, et al. Readmissions following inpatient treatment for opioid-related conditions. Subst Use Misuse. 2019;54(3):473-81. https://doi.org/10.1080/10826084.201 8.1517174 .

20. Calcaterra SL, Binswanger IA, Edelman EJ, McNair BK, Wakeman SE, O'Connor PG. The impact of access to addiction specialist on attitudes, beliefs and hospital-based opioid use disorder related care: a survey of hospitalist physicians. Subst Abus. 2020:1-9. https://doi.org/10.1080/ 08897077.2020.1748169.

21. Janjua NZ, Islam N, Kuo M, Yu A, Wong S, Butt ZA, et al. Identifying injection drug use and estimating population size of people who inject drugs using healthcare administrative datasets. Int J Drug Policy. 2018;55:31-9. https:// doi.org/10.1016/j.drugpo.2018.02.001.

22. Jacka B, Larney S, Degenhardt L, Janjua N, Høj S, Krajden M, et al. Prevalence of injecting drug use and coverage of interventions to prevent HIV and hepatitis C virus infection among people who inject drugs in Canada. Am J Public Health. 2020;110(1):45-50. https://doi.org/10.2105/A JPH.2019.305379.

23. Nordeck CD, Welsh C, Schwartz RP, Mitchell SG, Cohen A, O'Grady KE, et al. Rehospitalization and substance use disorder (SUD) treatment entry among patients seen by a hospital SUD consultation-liaison service. Drug Alcohol Depend. 2018;186:23-8. https://doi.org/10.1016/j.drugalcdep.2017.12.043.

24. D'Onofrio G, O'Connor PG, Pantalon MV, Chawarski MC, Busch SH, Owens $\mathrm{PH}$, et al. Emergency department-initiated buprenorphine/naloxone treatment for opioid dependence: a randomized clinical trial. JAMA. 2015; 313(16):1636-44. https://doi.org/10.1001/jama.2015.3474.

25. Bråbäck $M$, Ekström L, Troberg K, Nilsson S, Isendahl P, Brådvik L, et al. Malmö treatment referral and intervention study-high 12-month retention rates in patients referred from syringe exchange to methadone or buprenorphine/naloxone treatment. Front Psychiatr. 2017:8:161. https://doi. org/10.3389/fpsyt.2017.00161.

26. Englander H, King C, Nicolaidis C, Collins D, Patten A, Gregg J, et al. Predictors of opioid and alcohol pharmacotherapy initiation at hospital discharge among patients seen by an inpatient addiction consult service. J Addict Med. 2020;14(5):415-22. https://doi.org/10.1097/ADM. 0000000000000611.

27. Woolf $\mathrm{SH}$, Schoomaker $\mathrm{H}$. Life expectancy and mortality rates in the United States, 1959-2017. Jama. 2019;322(20):1996-2016. https://doi.org/10.1001/ja ma.2019.16932.

28. Friedman J, Beletsky L, Schriger DL. Overdose-related cardiac arrests observed by emergency medical services during the US COVID-19 epidemic. JAMA Psychiatr. 2020.

29. Crowley R, Kirschner N, Dunn AS, Bornstein SS. Health and public policy to facilitate effective prevention and treatment of substance use disorders involving illicit and prescription drugs: an American College of Physicians Position Paper. Ann Intern Med. 2017;166(10):733-6. https://doi.org/10.7326/ M16-2953.

30. Infectious Diseases Society of America; HIV Medicine Association; Pediatric Infectious Diseases Society. Infectious Diseases and Opioid Use Disorder (OUD) Policy Issues and Recommendations. https://www.idsociety.org/globa lassets/idsa/topics-of-interest/opioid/id-and-the-opioid-epidemic-policybrief_3-19-2018-updated.pdf. Published 2018. Accessed 28 Dec 2020.

31. Kuehn B. NIH strategy to combat opioid crisis. Jama. 2017;318(24):2418. https://doi.org/10.1001/jama.2017.19928.

32. Springer SA, Korthuis PT, Del Rio C. Integrating treatment at the intersection of opioid use disorder and infectious disease epidemics in medical settings: a call for action after a National Academies of sciences, engineering, and medicine workshop. Ann Intern Med. 2018;169(5):335-6. https://doi.org/10. 7326/M18-1203.

33. Herscher M, Fine M, Navalurkar R, Hirt L, Wang L. Diagnosis and Management of Opioid use Disorder in hospitalized patients. Med Clin North Am. 2020;104(4):695-708. https://doi.org/10.1016/j.mcna.2020.03.003.

34. Eaton EF, Vettese T. Management of Opioid use Disorder and Infectious Disease in the inpatient setting. Infect Dis Clin N Am. 2020;34(3):511-24. https://doi.org/10.1016/j.idc.2020.06.008.

35. Chutuape MA, Katz EC, Stitzer ML. Methods for enhancing transition of substance dependent patients from inpatient to outpatient treatment. Drug Alcohol Depend. 2001;61(2):137-43. https://doi.org/10.1016/S0376-871 6(00)00133-2.

36. Accreditation Council for Graduate Medical Education. Programs in Florida in Addiction Medicine and Addiction Psychiatry. https://apps.acgme.org/a ds/public/. Published 2020. Accessed 28 Dec 2020.

37. Nunes EV, Kunz K, Galanter M, O'Connor PG. Addiction psychiatry and addiction medicine: the evolution of addiction physician specialists. Am J Addict. 2020;29(5):390-400. https://doi.org/10.1111/ajad.13068.

38. Lister JJ, Weaver A, Ellis JD, Himle JA, Ledgerwood DM. A systematic review of rural-specific barriers to medication treatment for opioid use disorder in the United States. Am J Drug Alcohol Abuse. 2020;46(3):273-88. https://doi. org/10.1080/00952990.2019.1694536.

39. Florida Department of Children and Family Services. Florida's State Opioid Response Project. https://www.myflfamilies.com/service-programs/samh/ opioidSTRP.shtml Accessed 28 Dec 2020.

40. National Academies of Sciences Engineering and Medicine. Opportunities to Improve Opioid Use Disorder and Infectious Disease Services: Integrating Responses to a Dual Epidemic. Washington, DC: The National Academies Press; 2020.

\section{Publisher's Note}

Springer Nature remains neutral with regard to jurisdictional claims in published maps and institutional affiliations. 